ARTICLE

https://doi.org/10.1038/s41467-019-08389-6

\title{
Binder-free graphene oxide doughs
}

\author{
Che-Ning Yeh', Haiyue Huang ${ }^{1}$, Alane Tarianna O. Lim¹, Ren-Huai Jhang1,2, \\ Chun-Hu Chen (D) $^{1,2} \&$ Jiaxing Huang (1) ${ }^{1}$
}

Graphene oxide (GO) sheets have been used to construct various bulk forms of GO and graphene-based materials through solution-based processing techniques. Here, we report a highly cohesive dough state of GO with tens of weight percent loading in water without binder-like additives. The dough state can be diluted to obtain gels or dispersions, and dried to yield hard solids. It can be kneaded without leaving stains, readily reshaped, connected, and further processed to make bulk GO and graphene materials of arbitrary form factors and tunable microstructures. The doughs can be transformed to dense glassy solids of GO or graphene without long-range stacking order of the sheets, which exhibit isotropic and much enhanced mechanical properties due to hindered sliding between the sheets. GO dough is also found to be a good support material for electrocatalysts as it helps to form compliant interface to access the active particles.

\footnotetext{
${ }^{1}$ Department of Materials Science and Engineering, Northwestern University, Evanston, IL 60208, USA. ${ }^{2}$ Department of Chemistry, National Sun Yat-sen University, Kaohsiung 80424, Taiwan. Correspondence and requests for materials should be addressed to C.-H.C. (email: chunhu.chen@mail.nsysu.edu.tw) or to J.H. (email: jiaxing-huang@northwestern.edu)
} 
G raphene oxide (GO), an oxidative exfoliation product of graphite, has gained significant interest as a building block to create graphene-based (a.k.a., reduced graphene oxide, $\mathrm{r}$-GO) materials because of its excellent dispersibility in water and rich functionality ${ }^{1-6}$. GO dispersions are usually used as starting materials to construct various graphene-based architectures by solution-processing routes ${ }^{3,7-12}$. Direct fabrication of selfstanding GO structures from dispersions or gels has been largely limited to thin films $s^{3}$ and lightweight foams ${ }^{7,13}$. A dough is a highly cohesive, malleable, and viscoelastic state of matter that can be readily reshaped without fracture, which is very useful for making free-standing three-dimensional structures ${ }^{14,15}$. As is with many other particulate materials, a semi-solid state of GO can be obtained by using additives with binder-like functions $^{16-18}$, and additional processing steps are required if these additives need to be removed afterwards. It would be desirable to obtain ultrahigh concentration of GO (e.g., > $10 \mathrm{wt}$. \%) in water to see if a dough state is accessible without the need for binders or cross-linkers, which has been challenging. For example, one could in principle obtain concentration of GO in water by drying a dilute solution, or by re-hydrating a dried GO solid. Evaporation can remove water from dilute GO solutions, but it is difficult to obtain very thick and uniform GO dispersions owing to GO's tendency to go to the air-water surface, which hinders the evaporation process owing to their barrier properties ${ }^{19-21}$. On the other hand, adding small amounts of water to dried GO solids has also been difficult, because small aliquot of water tends to be absorbed locally, leading to non-uniform hydration. In addition, there had been some misunderstanding concerning the solubility of GO solids in water. It was once believed that GO papers, a very common form of GO solid made by filtration, are insoluble and cannot be re-dispersed in water ${ }^{3,22}$.
In an earlier work, we found that the insoluble GO papers obtained by filtrations are unintentionally cross-linked by the multivalent cationic contaminants released from some filter disks, and neat GO papers are indeed readily re-dispersed in water ${ }^{23}$ and can be glued together by water droplets ${ }^{24}$. It further suggests that the interlayer interactions between GO sheets can be weakened by water, and all neat GO structures should become dispersible in water. Based on this insight, here we report a "continuum" of GO-water mixtures, showing continuous transitions between four states, starting from a dilute dispersion, a thick gel, a malleable dough, and eventually to a dense solid as the water content decreases. This continuum finally completes the scope of GO-water mixtures with the long missing dough state, which typically has a mass fraction of $\mathrm{GO} \sim 10$ s of weight percent. GO doughs are found to be highly processable, can be shaped by common processing methods, and exhibit super extensibility ${ }^{25}$. This binder-free dough state of GO is a versatile material platform to fabricate bulk GO and graphene architectures with arbitrary shapes and tunable microstructures, including porosity and sheet alignment.

\section{Results}

GO-water continuum. A system consisting of GO and water was prepared along a continuum of increasing concentration of GO, transitioning between four states starting with a dilute dispersion, a thick gel, a malleable dough, and a dense solid as shown in Fig. 1a-d. GO was synthesized by a modified Hummer's method $^{26-28}$. Powders of GO were obtained in the form of a filter cake after filtering a dispersion of purified sheets and drying in vacuum. Dilute dispersions ( $<2$ wt. \%) were typically made by dispersing small pieces of dried GO filter cake in water. Direct preparation of higher-concentration dispersions from dried GO
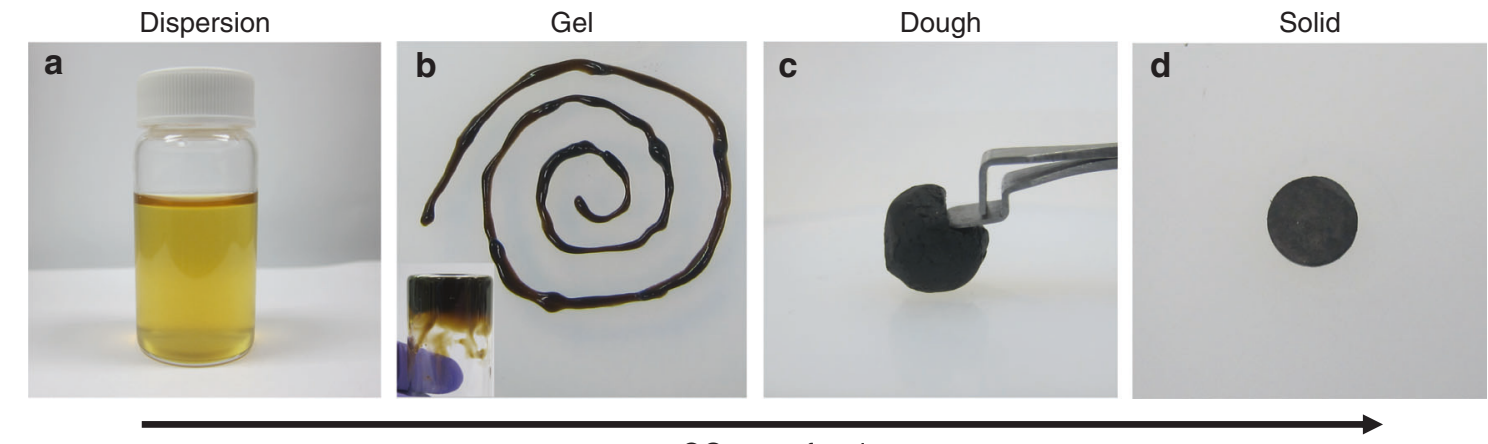

GO mass fraction
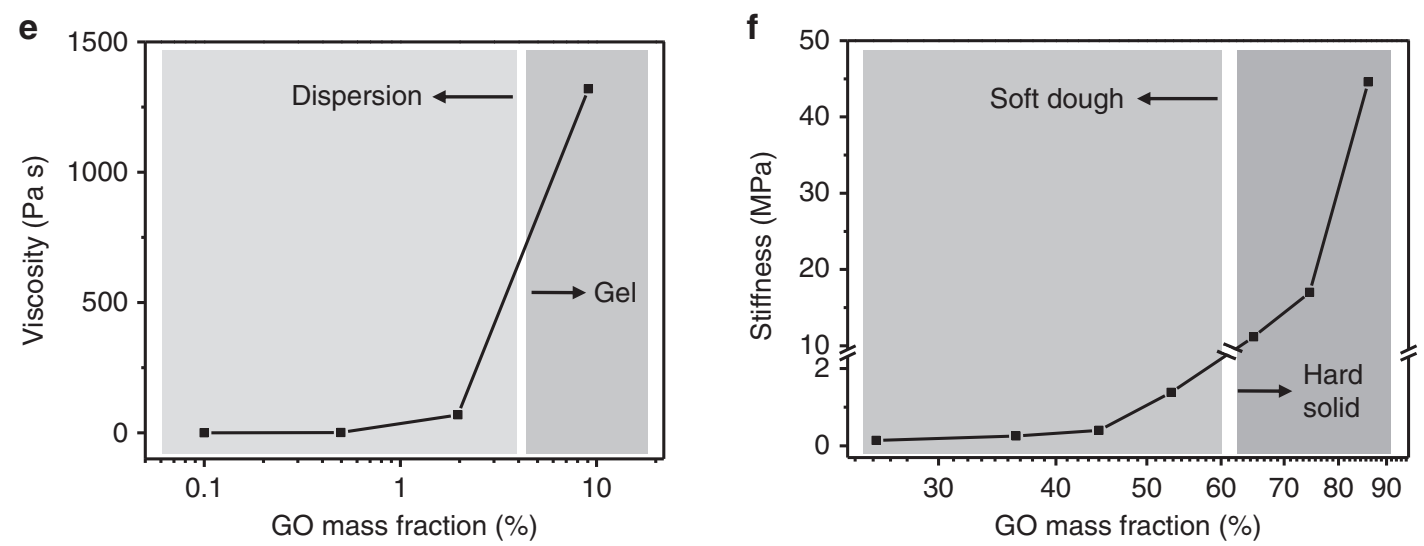

Fig. 1 The GO-water continuum. Photos showing GO-water mixture transitioning from a dilute dispersion, $\mathbf{b}$ a thick gel, $\mathbf{c}$ a malleable dough, to $\mathbf{d}$ a dense solid, as GO loading increases. e The transition from a dilute dispersion to a gel is characterized by a drastic increase in viscosity. $\mathbf{f}$ A dough-state GO is obtained when the mass fraction of GO exceeds $20 \mathrm{wt}$. \%. GO dough is highly malleable until its mass fraction is increased to over 60 wt. \%, after which the stiffness increases significantly 

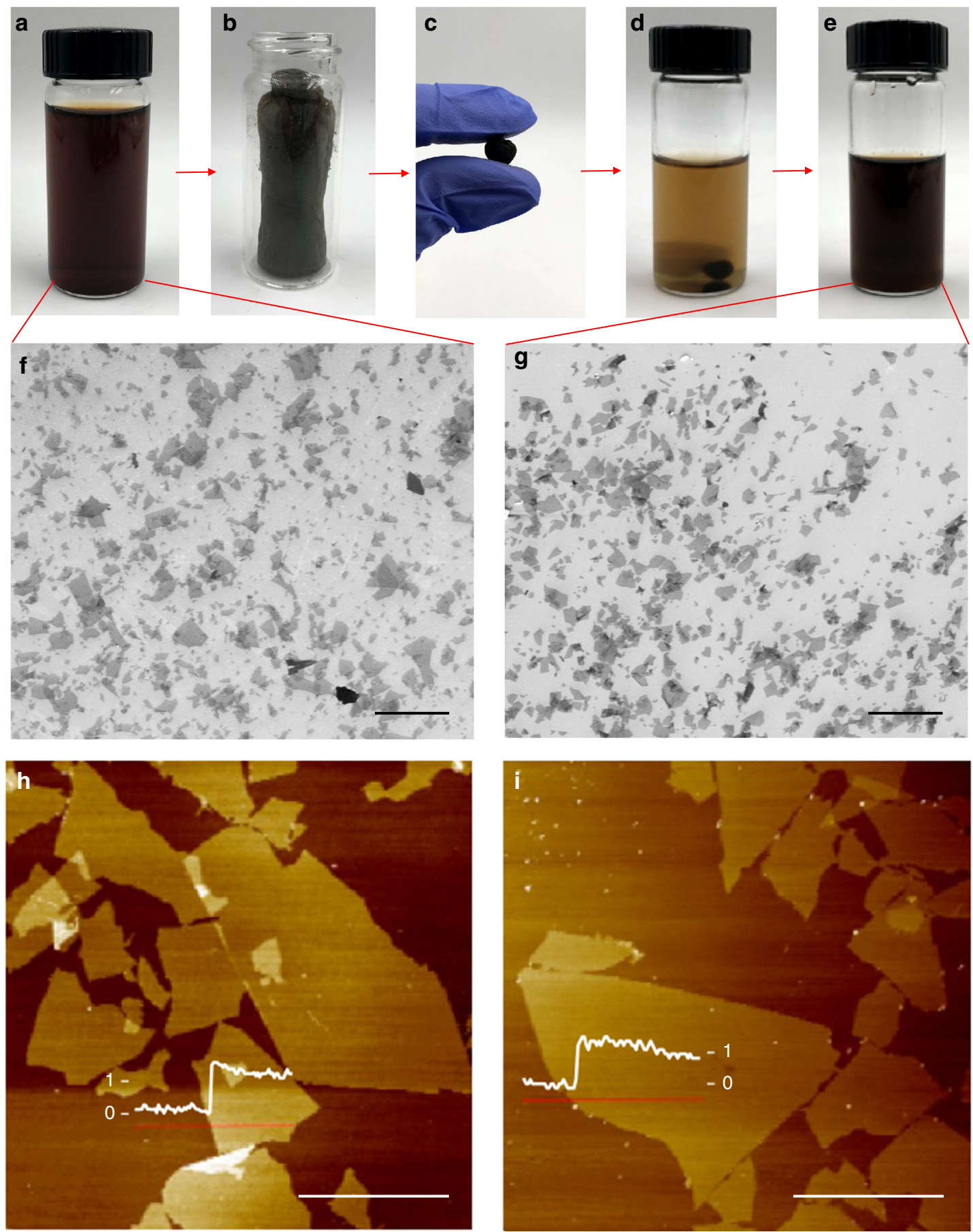

Fig. $2 \mathrm{GO}$ dough is readily re-dispersed in water. a-e Photos showing a GO sample converted from a dispersion to a foam, a dough, and back to the dispersion state. No significant changes in the sizes and morphology of GO sheets are observed before $\mathbf{f}, \mathbf{h}$ and after $\mathbf{g}$, $\mathbf{i}$ this process. $\mathbf{f}, \mathbf{g}$ (scale bar $=20 \mu \mathrm{m}$ ) and $\mathbf{h}, \mathbf{i}$ (scale bar $=10 \mu \mathrm{m}$ ) are images taken by SEM and AFM, respectively. Insets in $\mathbf{h}$ and $\mathbf{i}$ show height profiles of GO sheets in the unit of nanometers

was difficult as the apparent volume of GO powders was already comparable or higher than the volume of water needed. Therefore, adding a small aliquot of water usually resulted in local, nonuniform hydration of $\mathrm{GO}$ powders. In order to obtain uniform GO-water mixtures with very high GO loadings, aerosolized water mists were applied to GO foams obtained by freeze-drying, which collapsed upon water uptake. This process allows uniform hydration of GO by a minute amount of water throughout the entire volume of the material, which has been difficult by other means. Further kneading and rolling of the collapsed foam turned the material into a dough state (Supplementary Figure 1). The dough state of GO can serve as a precursor to make highconcentration gels by dilution, or denser solids by drying.

Transitions between the four states were accompanied by significant changes in their rheological and mechanical properties. As the mass fraction of $\mathrm{GO}$ was increased, the dilute dispersion turned into a thick non-flowing gel as illustrated by the sample in an upside-down vial (Fig. 1b inset). Accordingly, a significant increase in viscosity was observed when the GO mass fraction exceeded 2 wt. \% (Fig. 1e). Increasing the GO fraction to 

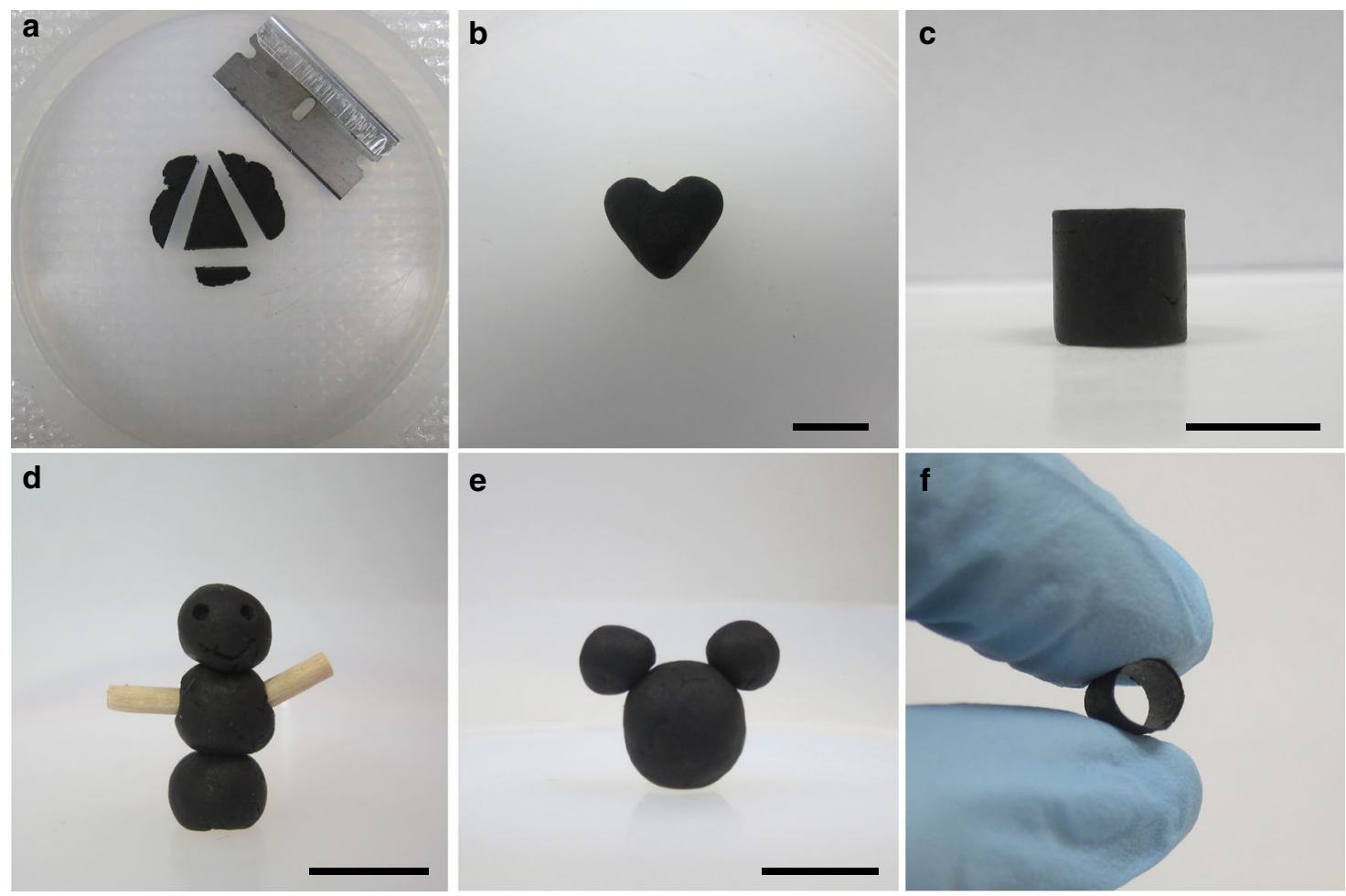

Fig. $3 \mathrm{GO}$ doughs are highly processable and versatile. GO doughs can be readily reshaped by a cutting, b pinching, c molding, and $\mathbf{d}$ carving. GO doughs can be easily connected together $\mathbf{d}$, e or with other solid materials $\mathbf{d}$ using the wooden sticks as an example. $\mathbf{f} A$ tubular $\mathrm{GO}$ structure can be prepared by molding a GO dough around a rod, demonstrating the versatility of using GO doughs to make 3D architectures that are otherwise challenging to obtain. Scale bars in $\mathbf{b}, \mathbf{c}, \mathbf{d}$, and $\mathbf{e}$ are $1 \mathrm{~cm}$

over $20 \mathrm{wt}$ \% resulted in a viscoelastic dough state. Unlike the thick gels, the dough state can be kneaded and rolled on a surface without leaving extensive stains. This dough state can be easily deformed without fracture, and dried to form a dense GO solid that retained the shape (Fig. 1d). The dough state remained highly cohesive and processable until the mass fraction reached $\sim 60$ wt. \%, after which the mixture became significantly stiffer, as characterized by a rapid increase of its compression modulus (Fig. 1f). As the loading of GO exceeded $60 \mathrm{wt}$. \%, the solid became fragile and tended to fracture after compression.

Results of viscosity measurements of the GO-water mixtures including dispersions and gels are shown in Supplementary Figure 2, showing shear thinning behaviors that can be attributed to shear alignment of sheets and reduced tangling at higher shear rates $^{29}$. Making the gel from the dough state is a lot more straightforward than other methods, and the rheological properties of the gels can be tuned over a large range, by simply adjusting the volume of water added. This allows customization of GO gels for a broad array of materials-processing techniques ${ }^{30}$, to create GO and graphene final products.

Binder-free GO dough: a compact form for storage and transportation. The dough state is a highly versatile form of GO that is ideal for manufacturing. The high mass loading and compact form factors of GO doughs make them much more economical to store and transport. And as GO doughs are free of binders or cross-linkers, they can be readily re-dispersed in water to re-generate high-quality GO dispersion. The photos in Fig. 2a-e depict the transition of a GO sample through different states, starting from a dispersion, a foam to a dough state, which is then placed in water to yield dispersion again. As shown in the scanning electron microscopy (SEM) and atomic force microscopy (AFM) images (Fig. $2 \mathrm{f}, \mathrm{h}$ ), the as-made GO sheets have lateral dimension of $\sim 5$ to $20 \mu \mathrm{m}$ and thickness of $1 \mathrm{~nm}$. The dispersion was then freeze dried to yield a foam (Fig. 2b), which was subsequently hydrated and kneaded to obtain the dough state (Fig. 2c). The dough was then placed in water (Fig. 2d), gently stirred for $\sim 10 \mathrm{mins}$, and sonicated in a tabletop sonicator for 2 mins, which results in a dispersion (Fig. 2e) with similar color, stability as the starting one (Fig. 2a). GO sheets spin coated from the final dispersion were found to have similar lateral dimension, thickness, and morphology (Fig. 2g, i) to these of starting GO sheets. These results suggest that converting GO dispersion into the much more compact dough state does not deteriorate the quality of GO sheets in terms of sizes and colloidal processability. Compared with other compact forms, such as dried powders or films, GO doughs are sufficiently hydrated to avoid spontaneous exothermal self-propagating reduction reaction ${ }^{27}$, and much safer to handle. GO doughs are also cleaner to handle than gels or pastes, owing to its high cohesivity and non-staining properties.

Moldability and extensibility of GO dough. The dough state of GO can be deformed into arbitrary form factors by common shaping methods, including cutting, shaping, molding, and carving (Fig. 3a-d). Pieces of GO doughs can be connected together simply by bringing them into contact followed by gentle compression (Fig. 3e). The versatility of GO dough allows unusual shapes to be made at ease, which has been hard by other means, as illustrated by the example of a tubular structure shown in Fig. 3f. As-made GO doughs isotropically shrinks upon air drying, yielding dense solids with disorderly packed sheets that are heavily wrinkled and crumpled (Fig. 4a-c). X-ray diffraction (XRD) pattern of the dried GO solid does not show an apparent peak $\sim 11^{\circ}$, which is characteristic for lamellar GO structures with long-range stacking order of the sheets (Fig. 4d). 

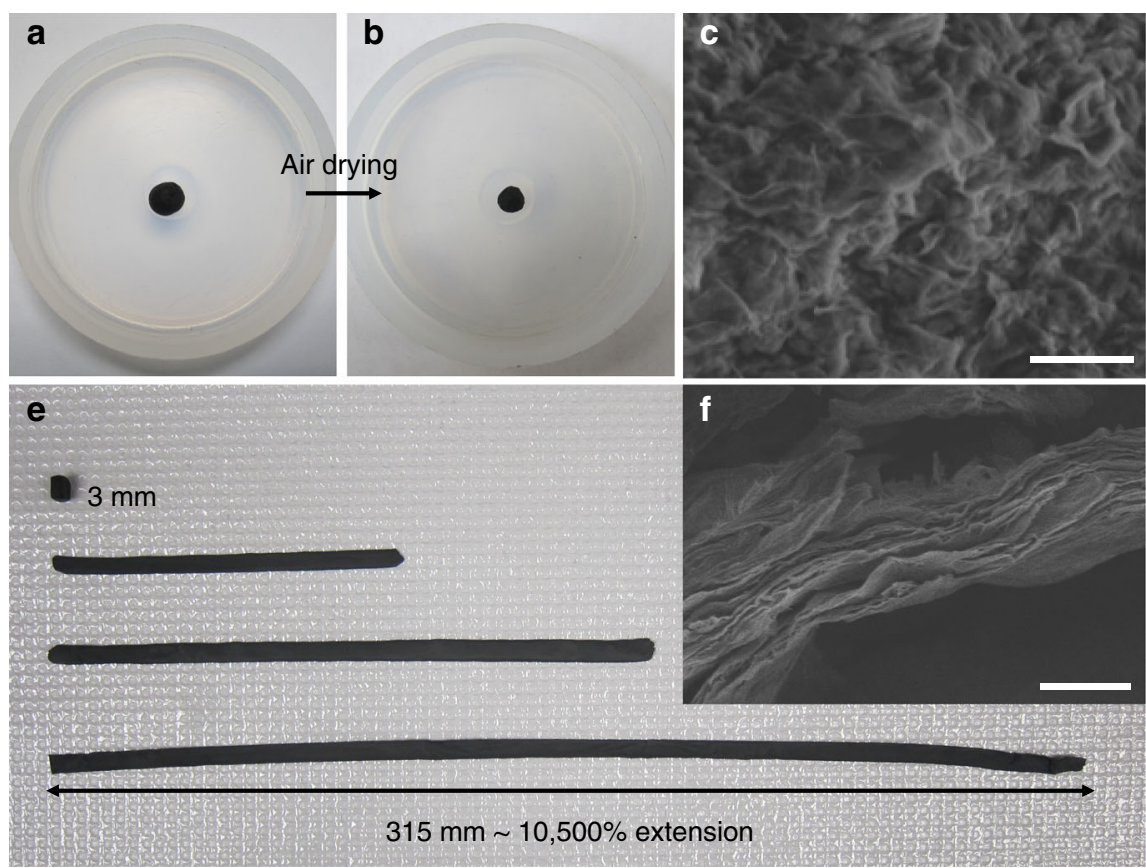
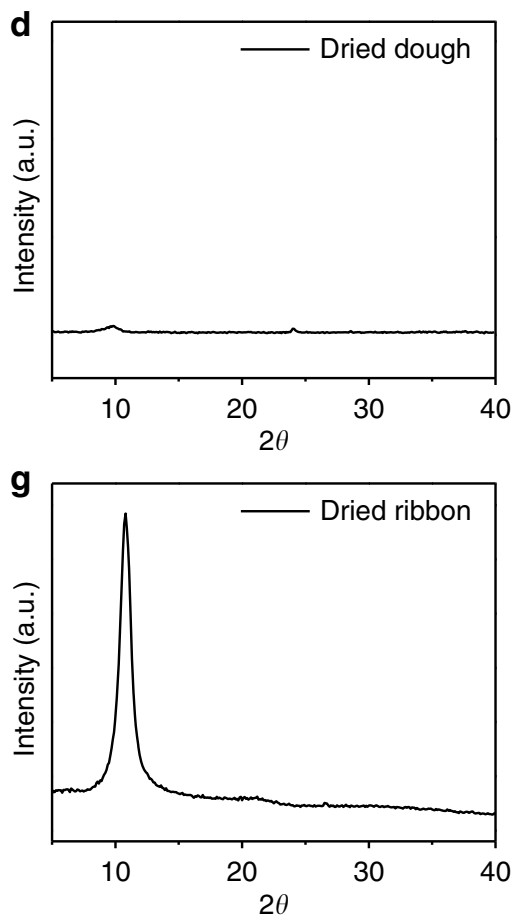

Fig. 4 Super extensibility of GO doughs. Photos of a ball-shaped GO dough a before and $\mathbf{b}$ after drying in air, showing slight volume shrinkage. c Crosssectional SEM image of the dried solid does not show lamellar microstructure, which is consistent with $\mathbf{d}$ the lack of a strong diffraction peak in the XRD pattern of the air-dried GO solid. e A short block of GO dough can be repeatedly cold rolled to a long $\mathrm{GO}$ ribbon with $10,500 \%$ of extension. $\mathbf{f}$ Crosssectional SEM image of the dried ribbon shows the development of lamellar microstructure, which is consistent with $\mathbf{g}$ the strong diffraction peak $\sim 11^{\circ}$ in the XRD pattern. Scale bar in $\mathbf{c}$ is $10 \mu \mathrm{m}$, and in $\mathbf{f}$ is $2 \mu \mathrm{m}$

The GO dough can sustain extreme deformation without fracture, exhibiting super extensibility. A proof-of-concept is shown in Fig. 4e, where a 3-mm-long GO block was transformed into a long ribbon by cold rolling with lateral constraint. Two additional rolling steps were performed to further increase the length to $315 \mathrm{~mm}$, corresponding to an elongation of $10,500 \%$. The extraordinary extensibility of the GO dough is attributed to both unfolding of the sheets and their sliding under shear. Indeed, the final cold-rolled ribbon has a lamellar microstructure (Fig. 4f). $\mathrm{XRD}$ pattern of the dried ribbon shows a strong diffraction peak, corresponding to an interlayer spacing of $8.18 \AA$, which is consistent with what has been reported for vacuum-filtered GO membranes $^{23}$ (Fig. 4g). Preparing thick GO films by vacuum filtration is quite tedious due to the barrier properties of the sheets that makes filtration process self-limiting. GO dough could thus serve as a more versatile starting material for creating largearea GO films by rolling with the thickness controllable by the gap between the rollers.

The dough state of GO can be extended to a number of GObased composites, leveraging GO's surfactant properties ${ }^{2,20}$ to incorporate other components. For example, single-walled carbon nanotubes (SWCNTs) can be readily dispersed in water in the presence of GO, which can then be converted into a GO/SWCNT composite dough following the same procedure (Supplementary Figure 3). This GO/SWCNT composite dough is still highly cohesive and processable, which can also be cold rolled into a free-standing membrane, suggesting the potential of preparing composite doughs with various combinations of $\mathrm{GO}$ and functional materials based on the preparation method provided in this work.

GO dough as a compliant support for electrocatalysts. The cohesivity, viscoelastic properties, and largely isotropic arrangement of sheets of GO dough makes it a suitable material platform to embed other particulate materials, such as fine particles for electrocatalytic purposes. Typically in electrocatalysis, catalytic particles need to be glued on electrodes with the help of binder materials (e.g., polymers or mineral oils) to improve adhesion, and conductive additives to improve charge transport (e.g., graphite and carbon black powders $)^{31-34}$. The roles of these additives can be fulfilled by GO dough, which may simplify the electrode preparation. Here the dough can be readily deformed to yield geometrically compliant interface with the embedded materials, which after reduction to $\mathrm{r}-\mathrm{GO}$, provides robust electrical contact. The largely isotropic arrangement of GO sheets in the dough state also facilitates mass transport during catalytic reactions. The cohesivity of the dough also helps to preserve electrode integrity during reactions.

A proof-of-concept experiment is carried out to compare the effectiveness of GO dough as a substrate for electrocatalytic oxygen evolution reactions (OER) with the conventional, widely used additive such as carbon paste or Nafion ${ }^{35}$. Commercial $\mathrm{RuO}_{2}$ particles are selected as the prototypical OER catalyst (see the details in Method), which was first embedded in a GO dough by direct mixing, followed by thermal annealing to convert GO to r-GO. As shown in Fig. 5a, the $\mathrm{r}-\mathrm{GO} / \mathrm{RuO}_{2}$ electrodes exhibit a small onset potential at $1.24 \mathrm{~V}$, which is very close to the theoretical limit of OER at $1.23 \mathrm{~V}$, suggesting highly efficient charge and mass transport enabled by the r-GO network to the active $\mathrm{RuO}_{2}$ particles. In contrast, the onset potentials for carbon paste $/ \mathrm{RuO}_{2}$ and Nafion/ $\mathrm{RuO}_{2}$ electrodes are $1.39 \mathrm{~V}$ and $1.41 \mathrm{~V}$, respectively. The electrochemical surface area ${ }^{36,37}$ results (Fig. 5b) show that the highest value of the GO dough based electrodes $\left(13.2 \mathrm{mF} \mathrm{cm}^{-2}\right)$ is about four times higher than those delivered by electrodes using carbon paste $\left(2.60 \mathrm{mF} \mathrm{cm}^{-2}\right)$ and Nafion binders $\left(2.91 \mathrm{mF} \mathrm{cm}^{-2}\right)$, indicating much higher accessibility to the active $\mathrm{RuO}_{2}$ powders in the r-GO monolith support. The SEM images 
a

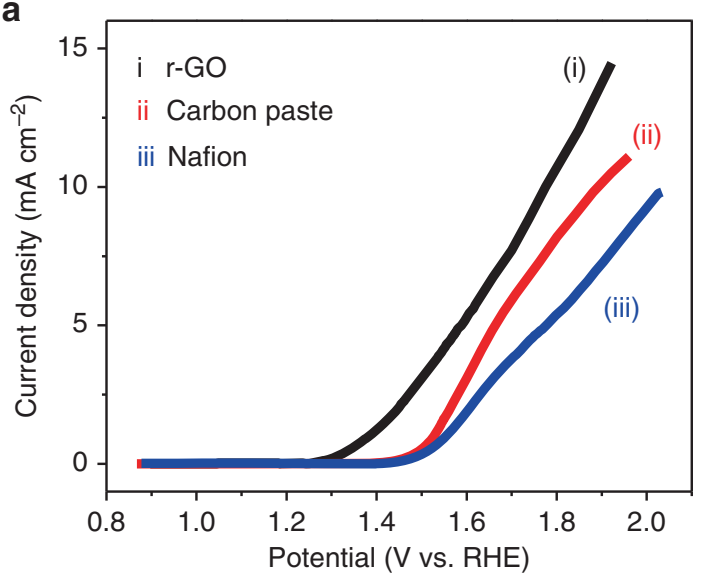

c
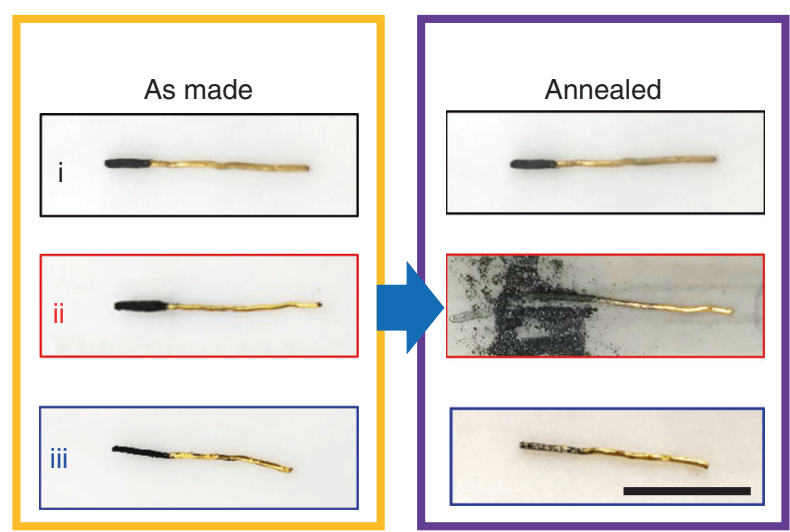

b

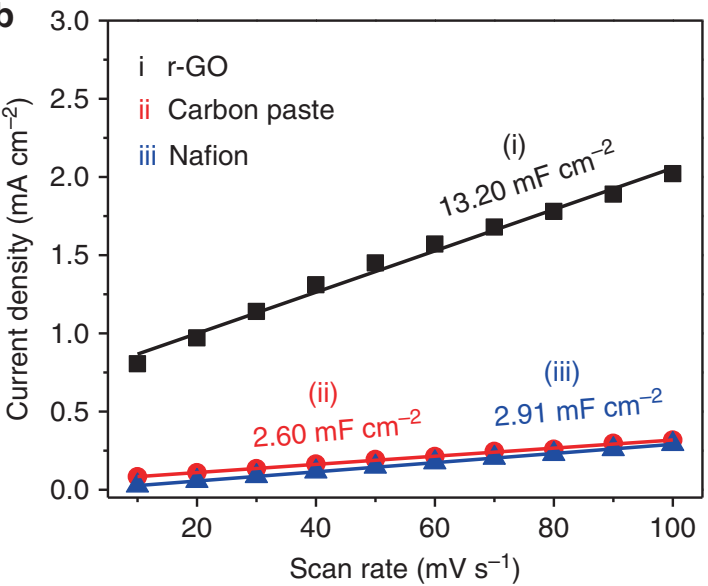

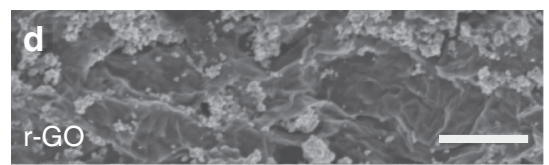
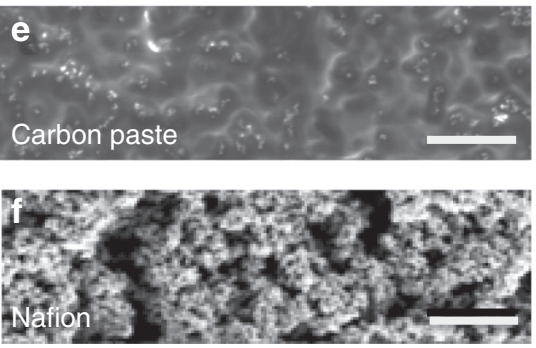

Fig. $5 \mathrm{GO}$ dough as a compliant support for electrocatalyst. a The linear sweep voltammetry curves of OER carried out in $0.1 \mathrm{M} \mathrm{KOH}$ using three different $\mathrm{RuO}_{2}$ electrodes made with annealed $\mathrm{GO}$ dough (i), carbon paste (ii), and Nafion (iii). b The electrochemical surface area of the three types of RuO 2 electrodes. c Photos showing the three types of electrodes before and after annealing. The $\mathrm{RuO}_{2}$ particles are fixed on gold wires through the three types of binders. d-f The SEM images showing the surface morphology of the three types of electrodes. Scale bar in $\mathbf{c}$ is $1 \mathrm{~cm}$, and in $\mathbf{d}$, e, and $\mathbf{f}$ are $5 \mu \mathrm{m}$

(Fig. $5 \mathrm{~d}-\mathrm{f}$ ) of the three types of electrodes confirmed that $\mathrm{RuO}_{2}$ particles are indeed more uniformly dispersed in the r-GO support.

Using GO dough as catalyst support expands the types of electrode form factors that can be readily obtained. In conventional electrode composition, the active catalytic particles are mixed together with other particulate additives; therefore, they are typically deposited on a metal substrate by casting. In contrast, the $\mathrm{r}-\mathrm{GO} / \mathrm{RuO}_{2}$ electrode can be directly pressed onto a metal contact from their dough state. For example, the electrode shown in Fig. 5c (i) was made by direct wrapping of a cold-rolled slice of $\mathrm{GO} / \mathrm{RuO}_{2}$ dough around a gold wire, followed by annealing. The resulting $\mathrm{r}-\mathrm{GO} / \mathrm{RuO}_{2}$ layer is quite robust, which not only sustained the annealing treatment, but also served as the efficient charge-transport contact for OER. In contrast, carbon paste and Nafion bound $\mathrm{RuO}_{2}$ electrodes tend to pulverize after annealing. Therefore, GO dough may be useful as a generic substrate for loading electrocatalyst particles for better evaluating their catalytic performance.

Glassy GO solids. The GO dough is a precursor to prepare dense GO solid with unique isotropic microstructure and properties. Typically, GO structures exhibit lamellar microstructures with long-range stacking order of GO sheets ${ }^{38}$, which leads to orientation dependent properties. However, as the dough is obtained by a small degree of hydration of GO foam, followed by kneading, the sheets are not aligned. During drying, the dough experiences isotropic capillary compression and gradually densifies by squeezing and deforming the GO sheets. The final microstructure is made of densely packed, heavily deformed sheets without apparent long-range stacking order, which bears strong resemblance to that of pistachio shells ${ }^{39}$. To study the effect of sheet alignment in the properties of dense GO solids, two bulk pellets with similar size, shape, and density but with different types of microstructures were prepared. One pellet was made by compressing a freeze-dried GO foam at $200 \mathrm{MPa}$ to induce alignment of the GO sheets (Fig. 6a, b). Such uniaxial compression indeed rendered the resulting pellet a lamellar microstructure (Fig. 6c) with a strong XRD peak corresponding to an interlayer spacing of $8.74 \AA$ (Fig. $6 \mathrm{~g}$ ). The other pellet was made by gently molding a GO dough into the same shape, followed by drying in air (Fig. 6d, e). Cross-sectional SEM image of the dried pellet did not show any obvious lamellar ordering (Fig. 6f), which is consistent with the lack of a strong diffraction peak $\sim 11^{\circ}$ in the XRD pattern (Fig. $6 \mathrm{~g}$ ). Both types of pellets have similar densities measured to be $\sim 1.5-1.6 \mathrm{~g} \mathrm{~cm}^{-3}$. The pellet with lamellar ordering is denoted as $l$-GO pellet, whereas the one with disordered sheets is denoted as glassy GO $(g-\mathrm{GO})$ pellet.

Indentation tests were applied to study the mechanical properties of the two types of pellets at both their top and side surfaces. The corresponding force-displacement curves are shown in Fig. $6 \mathrm{~h}$. In the $l$-GO pellet, the sheets are aligned in parallel to the top surface. Indentation on the side should encounter smaller resistance, because the load can easily cause sliding, deformation, or even partial opening of the lamellar 


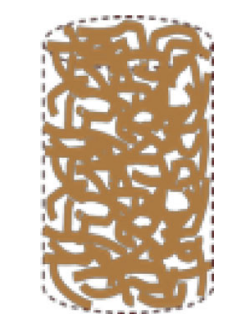

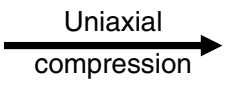

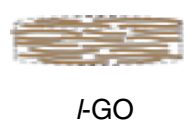

d
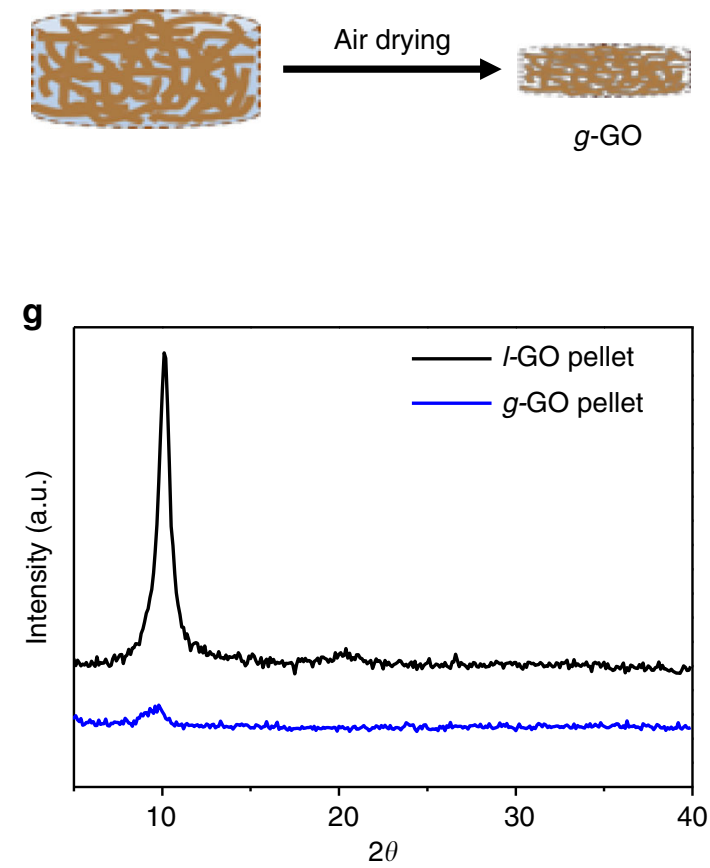
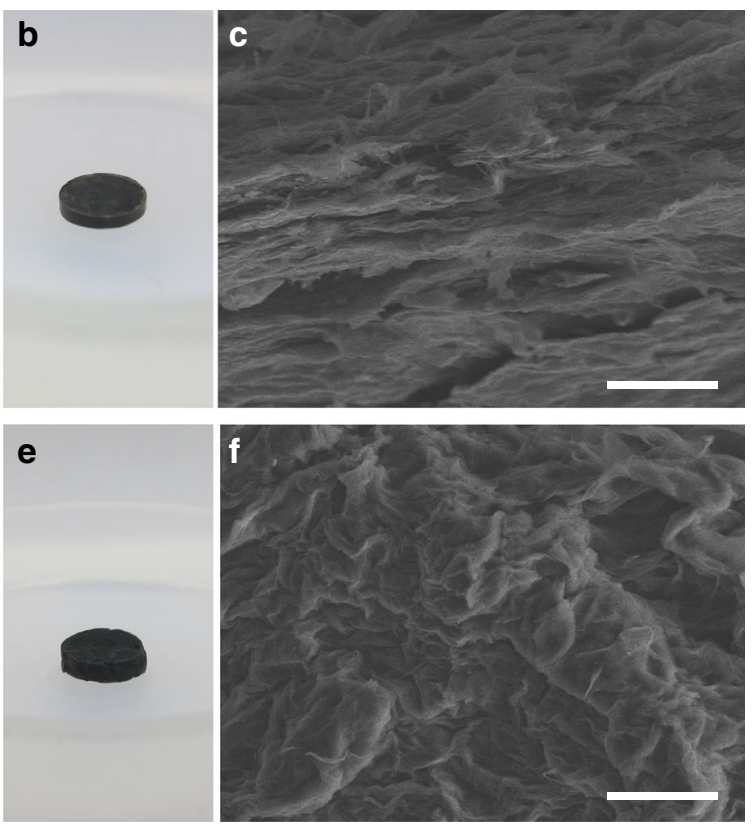

h

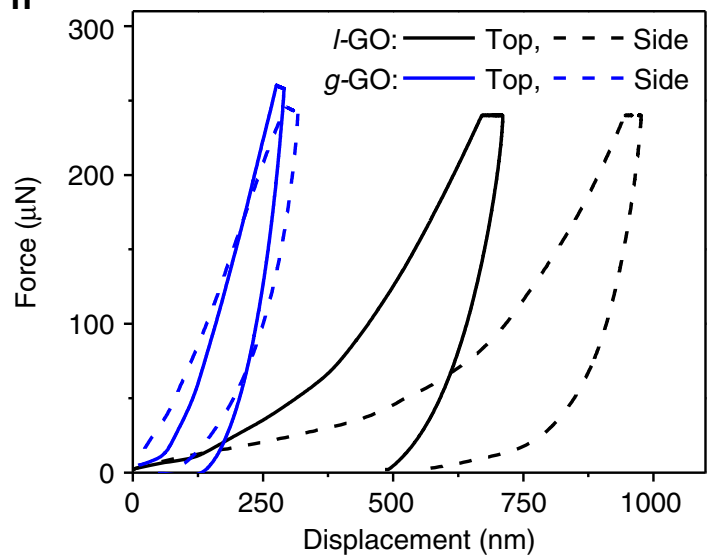

Fig. 6 Glassy GO solid with enhanced and isotropic mechanical properties. a-c Uniaxial compression turns a freeze-dried GO foam to a GO pellet with lamellar microstructure, which is denoted as I-GO. d-f A glassy GO pellet made of disorderly packed sheets, denoted as $g$-GO, is made by first gently molding the dough into a pellet by hand, followed by drying in air. $\mathbf{g}$ XRD patterns and $\mathbf{h}$ nanoindentation curves of the two types of GO pellets. The $g$-GO pellet does not have a strong diffraction peak corresponding to lamellar ordering, and exhibits largely isotropic properties and higher hardness. Scale bars in c and $\mathbf{f}$ are $10 \mu \mathrm{m}$

structure. Indentation along the top surface should encounter higher resistance, as the force is normal to the sliding direction of sheets. Indeed, the $l$-GO pellet is significantly softer on the side with a measured hardness of $13.38 \pm 3.02 \mathrm{MPa}$, in comparison with $25.29 \pm 5.20 \mathrm{MPa}$ measured on the top surface. Contrasting the anisotropic mechanical property of the l-GO pellet, the hardness of $g$-GO pellet does not exhibit significant orientation dependence, with a hardness of $133.37 \pm 14.76 \mathrm{MPa}$ measured on the top surface and $117.15 \pm 12.66 \mathrm{MPa}$ on the side. Similar displacement values were obtained when indenting the $g$-GO pellet from both its top surface and side. The hardness of $g$-GO pellet is drastically higher than those of $l$-GO. This is because the $g$-GO pellet is made of entangled and heavily crumpled sheets, which hinders sliding, making it much more resistant to deformation. Taken together, these results show that GO solids of similar size, shape, and density can exhibit significantly different properties owing to different sheet alignment. The GO dough is thus very suitable for creating bulk GO materials with isotropic properties.
Graphenic glass. As the dough state makes isotropic glassy GO solids readily accessible, it should also lead to glassy graphene solids after reducing GO. In such solids, the graphene sheets are densely but disorderly packed, exhibiting weak long-range stacking order. Therefore, they are named graphenic glass, taking inspirations from bulk metallic glass ${ }^{40}$. To prepare such glassy graphene solid, a GO dough (c.a., 50 wt. \%) was first molded into a cylindrical shape, and then reduced by HI vapor ${ }^{41}$, followed by washing with ethanol. The dried solid was further hot pressed under $50 \mathrm{MPa}$ at $800^{\circ} \mathrm{C}$ to a final density of $1.71 \mathrm{~g} \mathrm{~cm}^{-3}$ (Fig. 7a, inset), which is comparable to the densities of compressed graphite materials ${ }^{42}$. XRD pattern of the resulting graphenic solid only shows a very weak diffraction peak $\sim 26^{\circ}$, indicating the stacking of graphene sheets is rather disordered (Fig. 7c).

As is with glassy GO, sheet sliding is also significantly hindered in graphenic glass, which should make them harder than their lamellar counterpart. For comparison, a commercially available high strength graphite rod with a similar density of $1.78 \mathrm{~g} \mathrm{~cm}^{-3}$ (Fig. 7b, inset) was selected as a control sample. Such bulk 

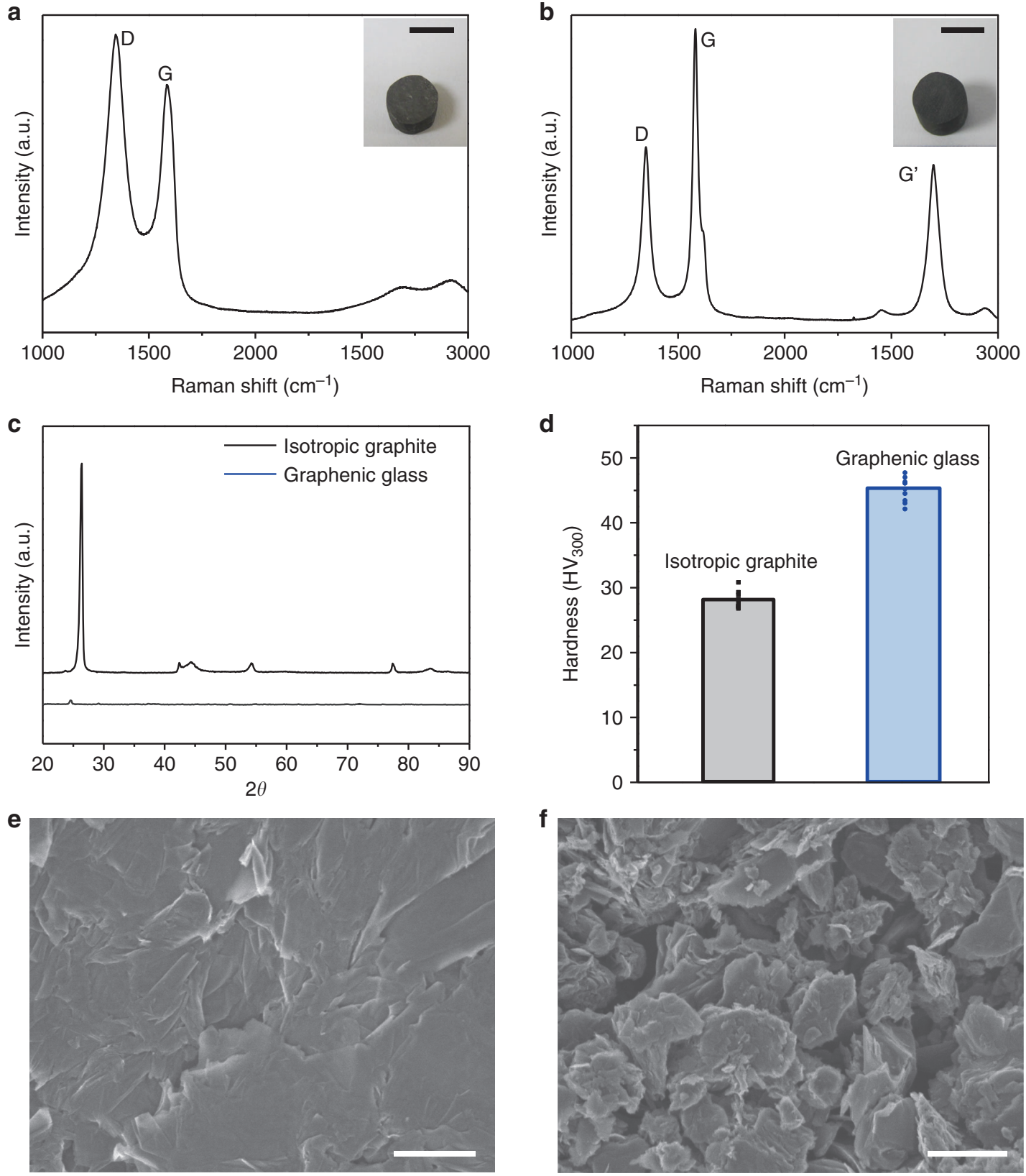

Fig. 7 Glassy graphene solid. Raman spectra of $\mathbf{a}$ a graphenic glass and $\mathbf{b}$ an isotropic graphite, showing that the graphenic glass is composed of more defective graphene sheets (insets of $\mathbf{a}, \mathbf{b}$ are photos of the two cylindrical samples). c XRD patterns show that the isotropic graphite is made of highly crystalline graphite grains while the graphenic glass, at similar density, does not have obvious long-range stacking order of the sheets. $\mathbf{d}$ Vickers hardness tests show that the graphenic glass is harder than the isotropic graphite, which can be attributed to $\mathbf{e}$ its smoother microstructure with finely distributed free volume (i.e., voids). In contrast, $\mathbf{f}$ the isotropic graphite has a much rougher and more granular microstructure, with much larger voids. Scale bars in a and $b$ insets are $5 \mathrm{~mm}$, and in $\mathbf{e}$ and $\mathbf{f}$ are $1 \mu \mathrm{m}$

graphite materials are typically made by isostatically densifying fine graphite powders with a binder, followed by calcination at elevated temperatures (e.g., $2500-3000^{\circ} \mathrm{C}$ ), and have widespread applications in areas including semiconductor industry and nuclear reactors ${ }^{43}$. Although the graphite grains still have anisotropic properties, the bulk solid is considered isotropic owing to the averaging effect of randomly oriented graphite grains. The isotropic graphite cylinder sample used in this work has relatively fine grains $\sim 5 \mu \mathrm{m}$, and is known for its comparatively high strength and hardness, which makes them suitable for making die sets for hot-pressing applications. In contrast, the glassy graphene solid is made of much weaker and less graphitized r-GO sheets. Indeed, Raman spectra (Fig. 7a, b) show that the $\mathrm{r}-\mathrm{GO}$ sheets in the glassy graphene solid is a lot more defective (with an $\mathrm{I}_{\mathrm{D}} / \mathrm{I}_{\mathrm{G}}$ ratio of 1.21) than those in the bulk graphite sample (with an $\mathrm{I}_{\mathrm{D}} / \mathrm{I}_{\mathrm{G}}$ ratio of 0.61 ) (Fig. $7 \mathrm{a}, \mathrm{b}$ ). Since the two samples have similar densities, and the isotropic graphite is much more graphitized and made of harder grains, one would expect the isotropic graphite should be harder than the graphenic glass. However, Vickers hardness tests show that the graphenic glass (with a hardness of $45.14 \pm 1.84 \mathrm{HV}_{300}$ ) is actually harder than the isotropic graphite (with a hardness of 28.01 \pm 1.29 $\mathrm{HV}_{300}$ ) (Fig. 7d). SEM images taken on their polished surfaces reveal drastically different microstructures. The graphenic glass has a relatively smooth texture, while the isotropic graphite shows a much rougher and more granular microstructure with visible 
voids (Fig. 7e, f). As the two samples have similar densities, they should have comparable level of free volume. Therefore, the smoother microstructure of the glassy graphene suggests that its free volume must be more finely divided and more uniformly distributed throughout the material. The higher hardness of the glassy graphene can be attributed to the finely distributed free volume and entangled network of the sheets within the solid, which renders it higher resistance to plastic deformation. In contrast, although the isotropic graphite has harder grains, it also has more segregated free volumes (i.e., voids), which cannot hinder sliding of the graphite grains, leading to lower hardness. The hardness of the graphenic glass is also higher than otherwise densified graphene materials including graphene-CNT hybrid solid made by pressure assisted welding 44 , and is comparable to that of the bulk graphene nanoplatelets fabricated by spark plasma sintering ${ }^{45}$. Dense graphene monoliths can be obtained from hydrogels made from hydrothermal reactions, and their size and shape are limited by the volume and shape of the hydrothermal reactors ${ }^{46-48}$. Therefore, the GO dough is a more versatile precursor to make bulk graphitic materials with arbitrary form factors, which does not rely on the use of binders, extensive mechanical compression, or high-temperature treatment.

\section{Discussion}

The GO-water continuum is now completed with a long missing dough state, which can be readily converted to thick gels by dilution or dense solids after drying. The dough state of GO is obtained without any binder and is highly processable. It can be deformed into arbitrary shapes and retains the shapes after drying. GO dough can act as highly compliant support for electrocatalytic particles, and after reduction to $\mathrm{r}-\mathrm{GO}$, offers high quality electrical interfaces to access the active catalyst during electrocatalysis. The dough can yield dense GO solids with disorderly packed sheets as a result of isotropic capillary compression during drying. Sheets in such glassy GO solids are crumpled and interlocked, leading to isotropic properties and higher hardness.

Glassy graphene solids can be made similarly after reducing GO sheets, which also exhibit isotropic properties. The graphenic glass, although made of less graphitized r-GO sheets, is even harder than commercial high-strength graphite materials owing to its unique microstructure limiting sheet sliding. This proof-ofconcept suggests the promise of the GO dough for fabricating high performance graphenic glass materials in the widespread applications of current bulk graphite materials.

The dough state of GO also brings some benefit to its storage and transportation during manufacturing owing to its high mass loading and compact form factors. GO doughs can also turn into high-quality dispersions simply by adding water. It is a safer form than dried powders or films ${ }^{27}$ owing to its higher degree of hydration and cohesivity, and a more convenient form to handle than gels and paste due to its non-staining properties. GO doughs can also be easily converted to dense GO or r-GO foams with tunable density and porosity. Their extraordinary processability can potentially make GO and graphene as easy to use as common engineering materials to create desirable size, shape, and structures in bulk forms ${ }^{49}$.

\footnotetext{
Methods

Preparation of GO dispersions. GO was synthesized using a modified Hummers method with a two-step purification process ${ }^{26-28}$. In a typical reaction, $6 \mathrm{~g}$ of graphite (Asbury, \#2139), $5 \mathrm{~g}$ of $\mathrm{K}_{2} \mathrm{~S}_{2} \mathrm{O}_{8}, 5 \mathrm{~g}$ of $\mathrm{P}_{2} \mathrm{O}_{5}$, and $25 \mathrm{~mL}$ of $\mathrm{H}_{2} \mathrm{SO}_{4}$ were stirred together at $80 \pm 5^{\circ} \mathrm{C}$. Next, the dispersion was diluted and filtered with filter papers (Whatman, Grade No. 3). The pre-treated graphite powders were then collected and dispersed in $230 \mathrm{~mL}$ of $\mathrm{H}_{2} \mathrm{SO}_{4}$. After that, $30 \mathrm{~g}$ of $\mathrm{KMnO}_{4}$ was slowly added. The mixture was kept at $35 \pm 5^{\circ} \mathrm{C}$ for $2 \mathrm{~h}$ and then slowly diluted with $0.5 \mathrm{~L}$ of deionised water, followed by the addition of $30 \mathrm{~mL}$ of $30 \% \mathrm{H}_{2} \mathrm{O}_{2}$. In the purification process, the mixture was first filtered using polytetrafluoroethylene
}

membrane (Millipore) and rinsed with $3.4 \% \mathrm{HCl}$ solution. After drying in vacuum, the as-prepared GO was re-dispersed and washed in acetone, and eventually filtered and dried again to yield GO cakes. The as-received GO cakes can be readily dispersed in water by shaking, which can be accelerated by gentle sonication (e.g., a few minutes in a tabletop sonicator). Solid chemicals were purchased from SigmaAldrich, and liquid chemicals were purchased from Fisher Chemical. All chemicals were used as received.

Preparation of freeze-dried GO foams. In a typical freeze-drying procedure, $10 \mathrm{~mL}$ of GO dispersions at a concentration of $5 \mathrm{mg} / \mathrm{mL}$ were prepared in glass vials. Such GO dispersions were then immersed in a liquid nitrogen bath to freeze for $10 \mathrm{~min}$. After GO dispersions were frozen, the vials were then transferred to a freezedryer, and porous and spongy GO foams were formed after the ice in frozen GO dispersions was completely sublimated (typically in 1-2 days).

Preparation of GO doughs. GO doughs were obtained by partially hydrating freeze-dried GO foams using water mist, which can be achieved by a handheld aerosol generator or blowing air through a hot water bath to carry moisture (Supplementary Figure 4). The color of the foam darkens upon hydration, which can be used to monitor degree of hydration. Kneading and rolling the hydrated GO foams yielded GO doughs with GO mass fractions generally between 40-60 wt. \% The degree of hydration can be further tuned by adjusting the amount of additional water added in the GO doughs.

From GO doughs to gels. Further hydrating the GO dough turned it into a sticky and gel-like material, which can be directly extruded via a syringe. The viscosity of the gel can be tuned at ease by adjusting the amount of water added into the dough.

Preparation of $\mathbf{r}-\mathbf{G O} / \mathbf{R u O} \mathbf{O}_{\mathbf{2}}$ electrode. $\mathrm{RuO}_{2}$ particles (Alfa Aesar) were blended in a GO dough (50 wt. \% GO content) using a mixer (Thinky AR-100) with a $\mathrm{RuO}_{2}$ : GO weight ratio of 3: 7. The resultant dough was cold rolled into a sheet and then wrapped around an $\mathrm{Au}$ wire $(0.5 \mathrm{~mm}$ in diameter). The diameter of the final electrode was $\sim 1 \mathrm{~mm}$. The $\mathrm{GO} / \mathrm{RuO}_{2}$ electrode was then freeze dried followed by thermal annealing at $250{ }^{\circ} \mathrm{C}$ for $2 \mathrm{~h}$ (heating rate: $1.5^{\circ} \mathrm{C} \mathrm{min}{ }^{-1}$ ) under argon to reduce GO. The $\mathrm{RuO}_{2}: \mathrm{r}-\mathrm{GO}$ weight ratio in the final electrodes is $\sim 3: 3.5$.

Preparation of carbon paste/ $/ \mathbf{R u O}_{2}$ electrode. Based on previously reported procedures ${ }^{31-33,50,51}$, carbon paste was prepared by mixing graphite powders (150 mesh) and mineral oil with a weight ratio of 70:30. Next $\mathrm{RuO}_{2}$ was added to the paste with a weight ratio of 3:3.5. The carbon/ $\mathrm{RuO}_{2}$ paste was then coated on an Au wire of $0.5 \mathrm{~mm}$ in diameter. Stability of the paste on the electrode was tested by annealing at $310^{\circ} \mathrm{C}$ under $\mathrm{Ar}$.

Preparation of Nafion/ $\mathbf{R u} \mathbf{O}_{\mathbf{2}}$ electrodes. Nafion was purchased from SigmaAldrich ( 5 wt. \% in water). The Nafion $/ \mathrm{RuO}_{2}$ electrode was prepared by mixing $4 \mathrm{mg} \mathrm{RuO}_{2}$ (i.e., identical to that used in the GO dough based electrodes) with $2 \mu \mathrm{L}$ of Nafion solution ( $0.5 \mathrm{wt}$. \% diluted by ethanol). The mixture was then coated on an $\mathrm{Au}$ wire $(0.5 \mathrm{~mm}$ in diameter), and dried at room temperature overnight. Stability of the Nafion/ $\mathrm{RuO}_{2}$ coating on Au was tested by annealing at $200{ }^{\circ} \mathrm{C}$ under $\mathrm{Ar}$.

Electrochemical measurements. The electrochemical tests were conducted using a three-electrode cell connected to an Autolab PGSTAT302N station. $\mathrm{RuO}_{2}$-coated $\mathrm{Au}$ wires were used as the working electrodes, a Pt plate was used as the counter electrode, and $\mathrm{Ag} / \mathrm{AgCl}$ as the reference electrode. Linear sweep voltammetry was conducted at a scan rate of $5 \mathrm{mV} \mathrm{s}^{-1}$ in $0.1 \mathrm{M} \mathrm{KOH}$. The potentials were transferred to reversible hydrogen electrode (RHE) via following equation:

$$
\mathrm{E}_{\mathrm{RHE}}=\mathrm{E}_{\mathrm{Ag} / \mathrm{AgCl}}+0.1976+0.059 \times \mathrm{pH}
$$

Electrochemical surface areas were measured using cyclic voltammetry from 1.025 to $1.125 \mathrm{~V}$ (vs. RHE) in $0.1 \mathrm{M} \mathrm{KOH}$. The scan rates were carried from 10 to $100 \mathrm{~mA} \mathrm{~s}^{-1}$ with an interval of $10 \mathrm{mV} \mathrm{s}^{-1}$. The slopes of current density (at $1.12 \mathrm{~V}$ ) as the function of scan rates were taken to be the double layer capacitance $\left(\mathrm{C}_{\mathrm{dl}}\right)$, which was multiplied by 1000 to calculate the electrochemical surface areas $\left(\mathrm{mF} \mathrm{cm}^{-2}\right)$.

Glassy GO solids. GO doughs were molded into arbitrary shapes such as a heart, a ball, and a pellet. Freeze-drying preserved their size and shape, leading to highdensity foams. Air drying or vacuum drying the GO doughs resulted in dense GO solids with isotropically packed sheets that also became crumpled. Such solids are named glassy GO solids due to lack of long-range stacking order reflected in their XRD patterns.

Preparation of lamellar and glassy GO pellets. High-density lamellar GO pellets were prepared by directly compressing freeze-dried GO foams in a stainless steel die at a pressure of $200 \mathrm{MPa}$. Glassy GO pellets were prepared from GO doughs, which were first molded into pellets by gentle pressing in a Teflon die by hand, 
followed by drying in air. The two pellets were fractured in order to study their mechanical properties perpendicular to the top surface of the pellets.

Graphenic glass. GO doughs with mass loading over $50 \mathrm{wt}$ \% were reduced using hydroiodic acid vapor ${ }^{41}$. GO doughs were put in a glass petri dish, and the petri dish was placed in a sealed beaker containing $1 \mathrm{~mL}$ of $\mathrm{HI}$ solution, which was then heated to $\sim 60^{\circ} \mathrm{C}$ for 2 days. The reduction process yielded r-GO solids with volume shrinkage. The $\mathrm{r}-\mathrm{GO}$ solids were then washed with ethanol, after which they were loaded in a graphite die and hot pressed at $800^{\circ} \mathrm{C}$ under a pressure of $50 \mathrm{MPa}$ for $10 \mathrm{~min}$. The heating rate was $5^{\circ} \mathrm{C} \mathrm{min}^{-1}$.

Isotropic graphite. Based on a survey of disclosed isotropic graphite properties, two isotropic graphite rods with higher strengths and hardness were purchased from MERSEN USA Greenville-MI Corp. and McMaster-Carr for hardness measurement. The sample from MERSEN USA Greenville-MI Corp. was measured to have a higher hardness, and was chosen as a control sample.

Characterization. Elemental analysis of the as-made GO was done by combustion elemental analysis (Atlantic Microlab, Norcross, GA), and the weight percentage of $\mathrm{C}, \mathrm{H}, \mathrm{Cl}$, and $\mathrm{S}$ were found to be $42.58 \%, 2.50 \%, 0.84 \%$, and $3.68 \%$, respectively. SEM images were obtained by field emission scanning electron microscopy (NOVA NanoSEM 600 and Hitachi SU8300). AFM images were acquired with a Park Systems XE-100 AFM under tapping mode. The XRD patterns were collected with a Rigaku Dmax powder diffractometer with $\mathrm{Cu}$ Ka radiation $(\lambda=1.5418 \AA)$ at $40 \mathrm{kV}$. Static mechanical uniaxial compressive tests were conducted with a dynamic mechanical analyzer (EltroForce 5500, BOSE). Rheological characterization was performed on a rheometer (Anton Paar, MCR 302) using a stainless steel coneand-plate geometry at room temperature. Hardness was obtained via nanoindentation (Hysitron TI 950 Triboindenter) using a standard three-sided pyramidal Berkovich probe with contact areas of $\sim 1-20 \mu \mathrm{m}^{2}$. As the grains of commercial isotropic graphite are bigger than the nanoindentation tip, its hardness was measured using Vickers hardness tests on a Duramin 5 (Struers) microhardness tester equipped with a square-based pyramidal diamond indenter. An indentation load of $300 \mathrm{gf}$ and a holding time of $10 \mathrm{~s}$ were applied with contact areas of $\sim 6000-12000 \mu \mathrm{m}^{2}$. Raman spectroscopy was performed with a laser beam wavelength of $532 \mathrm{~nm}$ (Acton TriVista CRS System).

\section{Data availability}

The data that support the findings of this study are available from the authors upon reasonable requests.

Received: 31 July 2018 Accepted: 7 January 2019

Published online: 24 January 2019

\section{References}

1. Eda, G. \& Chhowalla, M. Chemically derived graphene oxide: towards largearea thin-film electronics and optoelectronics. Adv. Mater. 22, 2392-2415 (2010).

2. Kim, J., Cote, L. J. \& Huang, J. X. Two dimensional soft material: new faces of graphene oxide. Acc. Chem. Res. 45, 1356-1364 (2012).

3. Dikin, D. A. et al. Preparation and characterization of graphene oxide paper. Nature 448, 457-460 (2007).

4. Li, D. \& Kaner, R. B. Materials science - graphene-based materials. Science 320, 1170-1171 (2008)

5. Loh, K. P., Bao, Q. L., Eda, G. \& Chhowalla, M. Graphene oxide as a chemically tunable platform for optical applications. Nat. Chem. 2, 1015-1024 (2010).

6. Zhu, Y., James, D. K. \& Tour, J. M. New routes to graphene, graphene oxide and their related applications. Adv. Mater. 24, 4924-4955 (2012).

7. Qiu, L., Liu, J. Z., Chang, S. L., Wu, Y. \& Li, D. Biomimetic superelastic graphene-based cellular monoliths. Nat. Commun. 3, 1241 (2012).

8. Becerril, H. A. et al. Evaluation of solution-processed reduced graphene oxide films as transparent conductors. ACS Nano 2, 463-470 (2008).

9. Xu, Z. \& Gao, C. Graphene chiral liquid crystals and macroscopic assembled fibres. Nat. Commun. 2, 571 (2011).

10. Tung, V. C., Allen, M. J., Yang, Y. \& Kaner, R. B. High-throughput solution processing of large-scale graphene. Nat. Nanotechnol. 4, 25 (2009).

11. Cote, L. J., Kim, F. \& Huang, J. X. Langmuir-Blodgett assembly of graphite oxide single layers. J. Am. Chem. Soc. 131, 1043-1049 (2009).

12. Akbari, A. et al. Large-area graphene-based nanofiltration membranes by shear alignment of discotic nematic liquid crystals of graphene oxide. Nat. Commun. 7, 12 (2016).

13. Zhu, C. et al. Highly compressible 3D periodic graphene aerogel microlattices. Nat. Commun. 6, 6962 (2015).
14. Chiou, K., Byun, S., Kim, J. \& Huang, J. Additive-free carbon nanotube dispersions, pastes, gels, and doughs in cresols. Proc. Natl. Acad. Sci. U. S. A 115, 5703-5708 (2018).

15. Oh, J. Y., Kim, S., Baik, H. K. \& Jeong, U. Conducting polymer dough for deformable electronics. Adv. Mater. 28, 4455-4461 (2016).

16. Jiang, Y. et al. Versatile graphene oxide putty-like material. Adv. Mater. 28, 10287-10292 (2016).

17. Li, Y. R., Chen, J., Huang, L., Li, C. \& Shi, G. Q. "Pottery" of porous graphene materials. Adv. Electron. Mater. 1, 7 (2015).

18. Shuyuan, L., et al. Synthetic multifunctional graphene composites with reshaping and self-healing features via a facile biomineralization-inspired process. Adv. Mater. 30, 1803004 (2018).

19. Krueger, M. et al. Drop-casted self-assembling graphene oxide membranes for scanning electron microscopy on wet and dense gaseous samples. ACS Nano 5, 10047-10054 (2011).

20. Kim, J. et al. Graphene oxide sheets at interfaces. J. Am. Chem. Soc. 132, 8180-8186 (2010).

21. Chen, C. et al. Self-assembled free-standing graphite oxide membrane. $A d v$. Mater. 21, 3007-3011 (2009).

22. Joshi, R. K. et al. Precise and ultrafast molecular sieving through graphene oxide membranes. Science 343, 752-754 (2014).

23. Yeh, C. N., Raidongia, K., Shao, J. J., Yang, Q. H. \& Huang, J. X. On the origin of the stability of graphene oxide membranes in water. Nat. Chem. 7, 166-170 (2015).

24. Luo, C., Yeh, C. N., Baltazar, J. M. L., Tsai, C. L. \& Huang, J. X. A cut-andpaste approach to 3D graphene-oxide-based architectures. Adv. Mater. 30, 6 (2018).

25. Lu, L., Sui, M. \& Lu, K. Superplastic extensibility of nanocrystalline copper at room temperature. Science 287, 1463-1466 (2000).

26. Hummers, W. S. \& Offeman, R. E. Preparation of graphitic oxide. J. Am. Chem. Soc. 80, 1339-1339 (1958).

27. Kim, F. et al. Self-propagating domino-like reactions in oxidized graphite. Adv. Funct. Mater. 20, 2867-2873 (2010)

28. Luo, J., Kim, J. \& Huang, J. Material processing of chemically modified graphene: Some challenges and solutions. Acc. Chem. Res. 46, 2225-2234 (2013).

29. Kumar, P., Maiti, U. N., Lee, K. E. \& Kim, S. O. Rheological properties of graphene oxide liquid crystal. Carbon N. Y. 80, 453-461 (2014).

30. Søndergaard, R. R., Hösel, M. \& Krebs, F. C. Roll-to-roll fabrication of large area functional organic materials. J. Polym. Sci. B. 51, 16-34 (2013).

31. Huang, J., Wang, D., Hou, H. \& You, T. Electrospun palladium nanoparticleloaded carbon nanofibers and their electrocatalytic activities towards hydrogen peroxide and NADH. Adv. Funct. Mater. 18, 441-448 (2008).

32. Nohra, B. et al. Polyoxometalate-based metal organic frameworks (POMOFs): structural trends, energetics, and high electrocatalytic efficiency for hydrogen evolution reaction. J. Am. Chem. Soc. 133, 13363-13374 (2011).

33. Walcarius, A. Mesoporous materials and electrochemistry. Chem. Soc. Rev. 42 , 4098-4140 (2013).

34. Blasco-Ahicart, M., Soriano-Lopez, J., Carbo, J. J., Poblet, J. M. \& GalanMascaros, J. R. Polyoxometalate electrocatalysts based on earth-abundant metals for efficient water oxidation in acidic media. Nat. Chem. 10, 24-30 (2018).

35. Chen, W. Q. et al. Heterojunctions of silver-iron oxide on graphene for lasercoupled oxygen reduction reactions. Chem. Commun. 54, 7900-7903 (2018).

36. Long, X. et al. Metallic iron-nickel sulfide ultrathin nanosheets as a highly active electrocatalyst for hydrogen evolution reaction in acidic media. J. Am. Chem. Soc. 137, 11900-11903 (2015).

37. Zhang, H. et al. Active sites implanted carbon cages in core-shell architecture: highly active and durable electrocatalyst for hydrogen evolution reaction. ACS Nano 10, 684-694 (2016).

38. Joshi, R., Alwarappan, S., Yoshimura, M., Sahajwalla, V. \& Nishina, Y. Graphene oxide: the new membrane material. Appl. Mater. Today 1, 1-12 (2015).

39. Movva, M. \& Kommineni, R. Extraction of cellulose from pistachio shell and physical and mechanical characterisation of cellulose-based nanocomposites. Mater. Res. Expr. 4, 045014 (2017).

40. Miller, M. \& Liaw, P. Bulk metallic glasses: an overview (Springer Science \& Business Media, 2007).

41. Pei, S., Zhao, J., Du, J., Ren, W. \& Cheng, H.-M. Direct reduction of graphene oxide films into highly conductive and flexible graphene films by hydrohalic acids. Carbon N. Y. 48, 4466-4474 (2010).

42. Oku, T. \& Eto, M. Correlation between the strength and Vickers hardness of some nuclear graphite. Carbon N. Y. 12, 477-479 (1974).

43. Somiya, S. Handbook of advanced ceramics: materials, applications, processing, and properties (Academic press, 2013).

44. Nautiyal, P., Embrey, L., Boesl, B. \& Agarwal, A. Multi-scale mechanics and electrical transport in a free-standing 3D architecture of graphene and carbon nanotubes fabricated by pressure assisted welding. Carbon N. Y. 122, 298-306 (2017). 
45. Nieto, A., Lahiri, D. \& Agarwal, A. Synthesis and properties of bulk graphene nanoplatelets consolidated by spark plasma sintering. Carbon N. Y. 50, 4068-4077 (2012).

46. $\mathrm{Bi}, \mathrm{H}$. et al. Low temperature casting of graphene with high compressive strength. Adv. Mater. 24, 5124-5129 (2012).

47. Worsley, M. A. et al. Toward macroscale, isotropic carbons with graphenesheet-like electrical and mechanical properties. Adv. Funct. Mater. 24, 4259-4264 (2014).

48. Tao, Y. et al. Towards ultrahigh volumetric capacitance: graphene derived highly dense but porous carbons for supercapacitors. Sci. Rep. 3, 2975 (2013).

49. Luo, J., Gao, J., Wang, A. \& Huang, J. Bulk nanostructured materials based on two-dimensional building blocks: a roadmap. ACS Nano 9, 9432-9436 (2015).

50. Adams, R. N. Carbon paste electrodes. Anal. Chem. 30, 1576-1576 (1958).

51. Bobbitt, J. M., Colaruot, J. F. \& Huang, S. J. Preparative graphite-paste electrode. J. Electrochem. Soc. 120, 773-773 (1973).

\section{Acknowledgements}

The work was mainly supported by the Office of Naval Research (ONR N000141612838). A.T.O.L. thanks the National Science Foundation for a Graduate Research Fellowship. C.-N.Y. acknowledges $3 \mathrm{M}$ for a graduate fellowship, International Institute for Nanotechnology at Northwestern University for a Ryan Fellowship, and Robert R. McCormick School of Engineering and Applied Science at Northwestern University for a Terminal Year Fellowship. R.-H.J. and C.-H.C. thank the National Sun Yat-sen University to support their research visit at Northwestern, and the support from the Ministry of Science and Technology, Taiwan (107-2113-M-110-003 and 107-2918-I-110-004). This work made use of the EPIC facility and the SPID facility of Northwestern University's NUANCE Center, the Jerome B. Cohen X-Ray Diffraction Facility, and the MatCI Facility supported by the Soft and Hybrid Nanotechnology Experimental (SHyNE) Resource (NSF ECCS-1542205) and the MRSEC program (NSF DMR1720139) at Northwestern University. We also thank G. J. Snyder's group for use of their hot press, and Prof. SonBinh T. Nguyen, Dr. Jun Gao, Luke Prestowitz for helpful discussions.

\section{Author contributions}

J.H. conceptualized the dough state of GO and the resulting glassy state of bulk GO and graphene solids. All authors contributed to the design of the experiments, analysis of the results, and writing of the manuscript with R-H.J. and C-H.C. mainly responsible for the electrocatalysis work, and C-N.Y., H.H., and A.T.O.L. mainly responsible for the other parts.

\section{Additional information}

Supplementary Information accompanies this paper at https://doi.org/10.1038/s41467019-08389-6.

Competing interests: The authors declare no competing interests.

Reprints and permission information is available online at http://npg.nature.com/ reprintsandpermissions/

Journal peer review information: Nature Communications thanks Siegfried Eigler and the other anonymous reviewers for their contribution to the peer review of this work.

Publisher's note: Springer Nature remains neutral with regard to jurisdictional claims in published maps and institutional affiliations.

(c) (i) Open Access This article is licensed under a Creative Commons Attribution 4.0 International License, which permits use, sharing, adaptation, distribution and reproduction in any medium or format, as long as you give appropriate credit to the original author(s) and the source, provide a link to the Creative Commons license, and indicate if changes were made. The images or other third party material in this article are included in the article's Creative Commons license, unless indicated otherwise in a credit line to the material. If material is not included in the article's Creative Commons license and your intended use is not permitted by statutory regulation or exceeds the permitted use, you will need to obtain permission directly from the copyright holder. To view a copy of this license, visit http://creativecommons.org/ licenses/by/4.0/

(C) The Author(s) 2019 